\title{
Overview of Theory and Simulations in the Heavy Ion Fusion Science Virtual National Laboratory
}

\author{
Alex Friedman* \\ Lawrence Livermore National Laboratory, \\ PO Box 808, Livermore CA 94550 USA; \\ and Heavy Ion Fusion Science Virtual National Laboratory
}

(Dated: July 3, 2006)

Abstract

\begin{abstract}
The Heavy Ion Fusion Science Virtual National Laboratory (HIFS-VNL) is a collaboration of Lawrence Berkeley National Laboratory, Lawrence Livermore National Laboratory, and Princeton Plasma Physics Laboratory. These laboratories, in cooperation with researchers at other institutions, are carrying out a coordinated effort to apply intense ion beams as drivers for studies of the physics of matter at extreme conditions, and ultimately for inertial fusion energy. Progress on this endeavor depends upon coordinated application of experiments, theory, and simulations. This paper describes the state of the art, with an emphasis on the coordination of modeling and experiment; developments in the simulation tools, and in the methods that underly them, are also treated.
\end{abstract}

PACS numbers: 29.27.-a; 52.65.Cc; 52.65.Rr

Keywords: Accelerator, Fusion, Heavy-ion, Induction, Simulation, Particle-in-cell, Plasma, Beam

${ }^{*}$ Correspondence to: Dr. Alex Friedman, Mail Stop 47R0112, Lawrence Berkeley National Laboratory, One Cyclotron Road, Berkeley CA 94720; 510-486-5592; mailto:af@llnl.gov 


\section{INTRODUCTION}

The Heavy Ion Fusion Science Virtual National Laboratory (HIFS-VNL) is a collaboration of Lawrence Berkeley National Laboratory, Lawrence Livermore National Laboratory, and Princeton Plasma Physics Laboratory. These laboratories, in cooperation with researchers at other institutions, are carrying out a coordinated effort to apply intense ion beams as drivers for studies of matter at extreme conditions - in the Warm Dense Matter (WDM) regime, and ultimately at higher temperatures in the High Energy Density Physics (HEDP) regime, as expected in targets for Inertial Fusion Energy (IFE). Progress depends upon the coordinated use of experiments, theory, and simulations. This paper summarizes, and places in context, recent advances in model development and application. For an earlier treatment, see [1]. For an overview of the U.S. program in its entirety, see [2]. For a discussion of important related modeling on beam-target interaction and upcoming WDM physics experiments, see [3].

The simulation tools for modeling intense ion beams have enjoyed considerable development over the past several years. This paper describes the state of the art, with an emphasis on the coordination of modeling and experiment; developments in the tools themselves, and in the novel methods that underly them, are also treated. This paper is organized as follows: Section II describes the use of modeling to design and support experiments being conducted and planned in the HIFS-VNL. These include studies of neutralized compression and focusing; the Pulse Line Ion Accelerator concept; electron cloud and gas physics; solenoid transport; and injectors for both beams and plasmas. Section III describes progress on fundamental beam physics. Section IV briefly describes the application of these tools to other experimental programs, and other developments in the modeling tools. Section V offers comments on likely future directions.

\section{HIFS-VNL BEAM EXPERIMENTS}

\section{A. Neutralized Compression and Focusing}

Neutralized Drift Compression: In this process a velocity gradient ("tilt") is imposed on the beam, which then compresses as it drifts toward the target. For IFE, unneutralized compression has been assumed [4]. For WDM studies, which need shorter pulses, the beam must be neutralized during compression; this may prove advantageous for IFE as well. In recent experiments $[5,6]$ on the NDCX facility at LBNL, a carefully developed tilt waveform yielded sixty-fold longitudinal compression. Figure 1 shows the evolution of the longitudinal compression factor, as measured using the fast Faraday cup [7] and as simulated using the LSP code, a kinetic model, and a hybrid model [8]. The modeling shows that the compression achieved to date has been limited by known tilt waveform errors that can be further reduced; an improved pulser is planned. 


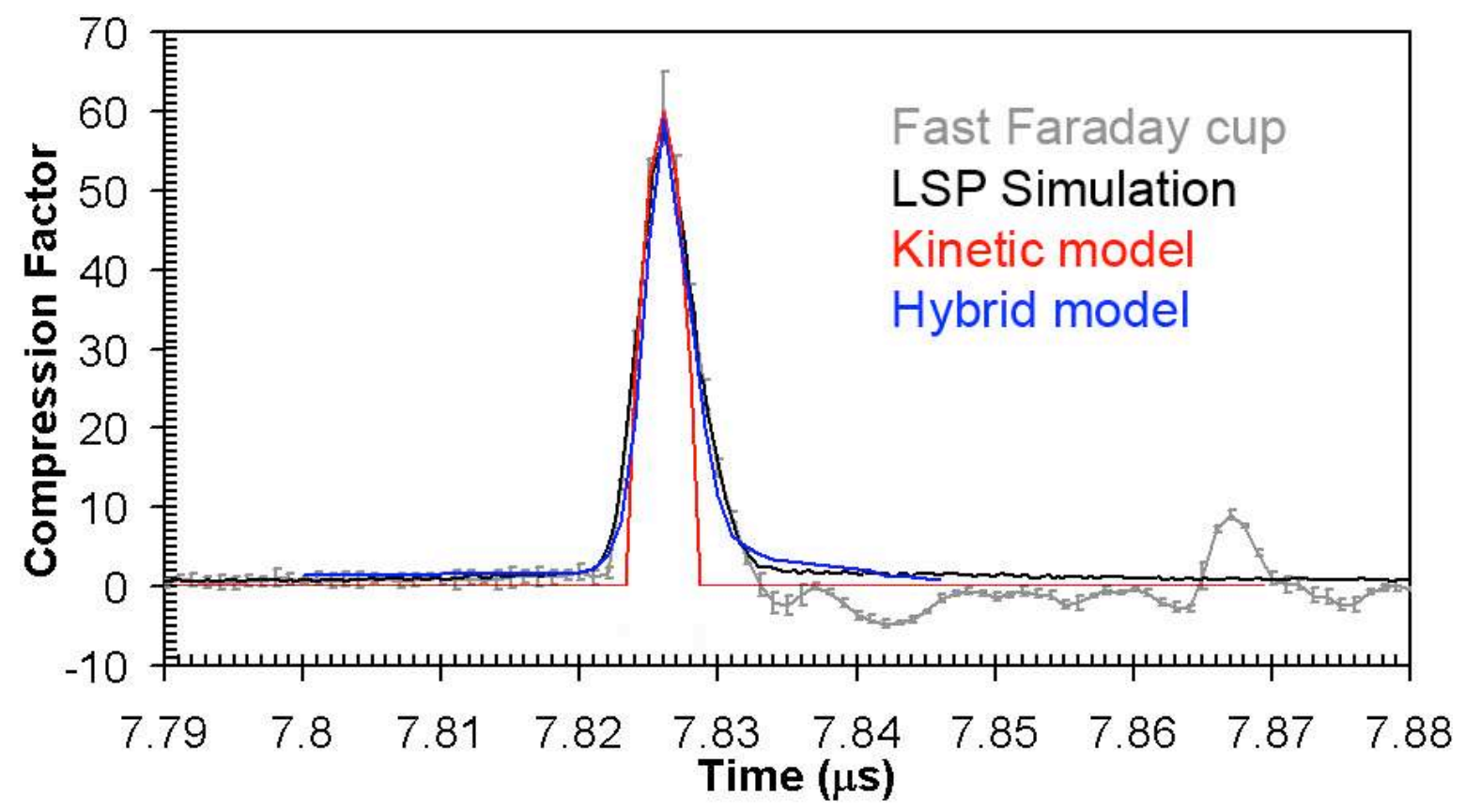

FIG. 1: Compression factor vs. time, as measured on NDCX, and as simulated using models indicated.

Combined compression and focusing: For both WDM and IFE applications, to be focused the beam must be neutralized as it passes through the target chamber and onto the target; this was studied in the earlier Neutralized Transport Experiment (NTX), and with simulations $[9,10]$. Experiments exploring the combination of neutralized drift compression and transverse focusing are planned for the near future. The factors limiting the ultimate intensity on target are errors in the voltage waveform applied to the "tilt gap"; time-dependent lens effects due to the fields in that gap; the longitudinal beam temperature $T_{L}$; and imperfect neutralization. The shortest (few-ns) pulses observed to date imply that on the NDCX apparatus $T_{L}<1.5 \mathrm{eV}$. Key issues being treated by the modeling include the ability to inject plasma of density at least equal to the beam density; the effectiveness of the dipole "trap" at preventing plasma flow upstream of the neutralized drift compression region; the transition from Brillouin or other unneutralized flow to neutralized transport; and control of beam-plasma interactions (including instabilities and wave excitation in the plasma) and stripping in the plasma column. Simulations using both WARP and LSP are underway to clarify the factors that influence $T_{L}$.

In one possible set of experiments on NDCX, a 15-T solenoid at the end of the drift region would focus the $0.08 \mathrm{~A}, 0.4 \mathrm{MeV}$ beam to a spot just as the line charge peaks. LSP runs showed a sub-millimeter focal spot and a peak beam density exceeding 
$10^{14} \mathrm{~cm}^{-3}$. Other runs examined use of the HCX beamline, with $0.36 \mathrm{~A}$ of $1.6 \mathrm{MeV}$ ions. Still others examined the source-to-target behavior of the beam on STX (the Solenoid Transport Experiment on NDCX); these confirmed the earlier runs in which the beam parameters upstream of the tilt core were supplied as input. See [11, 12].

NDC leaves the beam with a large velocity spread at final focus, which must be accommodated. Possible approaches include use of a strong solenoid, a plasma lens, and/or a plasma channel pinch, alone or in combination. A solenoid with graded field strength may provide adiabatic matching from the upstream line. Upstream correction using pulsed lenses (time-dependent focusing) is also possible; see [13] and the articles by Lee, Chen, and Yu in [14].

\section{B. Pulse Line Ion Accelerator}

For HEDP and WDM applications, the beam entering the accelerator is, ideally, shorter in duration, with a higher line-charge density, than has been assumed for IFE purposes. Suitable accelerator approaches were identified at a workshop [14, 15]; these included a new invention, the Pulse-Line Ion Accelerator (PLIA) [16]. The PLIA (Fig. 2) is essentially a helical distributed transmission line (a slow-wave structure). When driven by a smooth waveform, it operates in a long-wavelength, low-dispersion regime in which voltage pulses travel almost undistorted. A rising pulse applied to the upstream end appears at any instant as a spatial voltage ramp; a moving region contains an accelerating field along with which an ion pulse can travel and be steadily accelerated. The increase in ion energy can greatly exceed the applied voltage. The system is expected to be inexpensive, and favors short pulses. Further voltage multiplication can be obtained by coupling in the drive via a primary winding wrapped around the front of the helix.

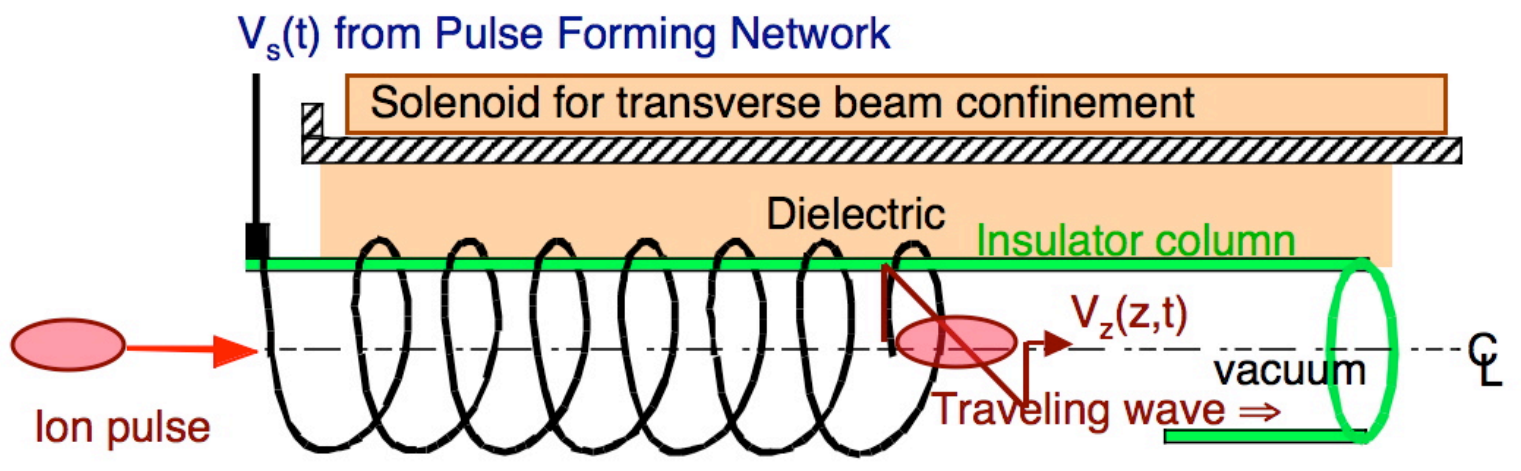

FIG. 2: Sketch of Pulse Line Ion Accelerator.

A lumped-element circuit model can describe wave behavior on the helix; elements include the capacitance of the helix to ground, the inter-turn capacitances, and the self- 


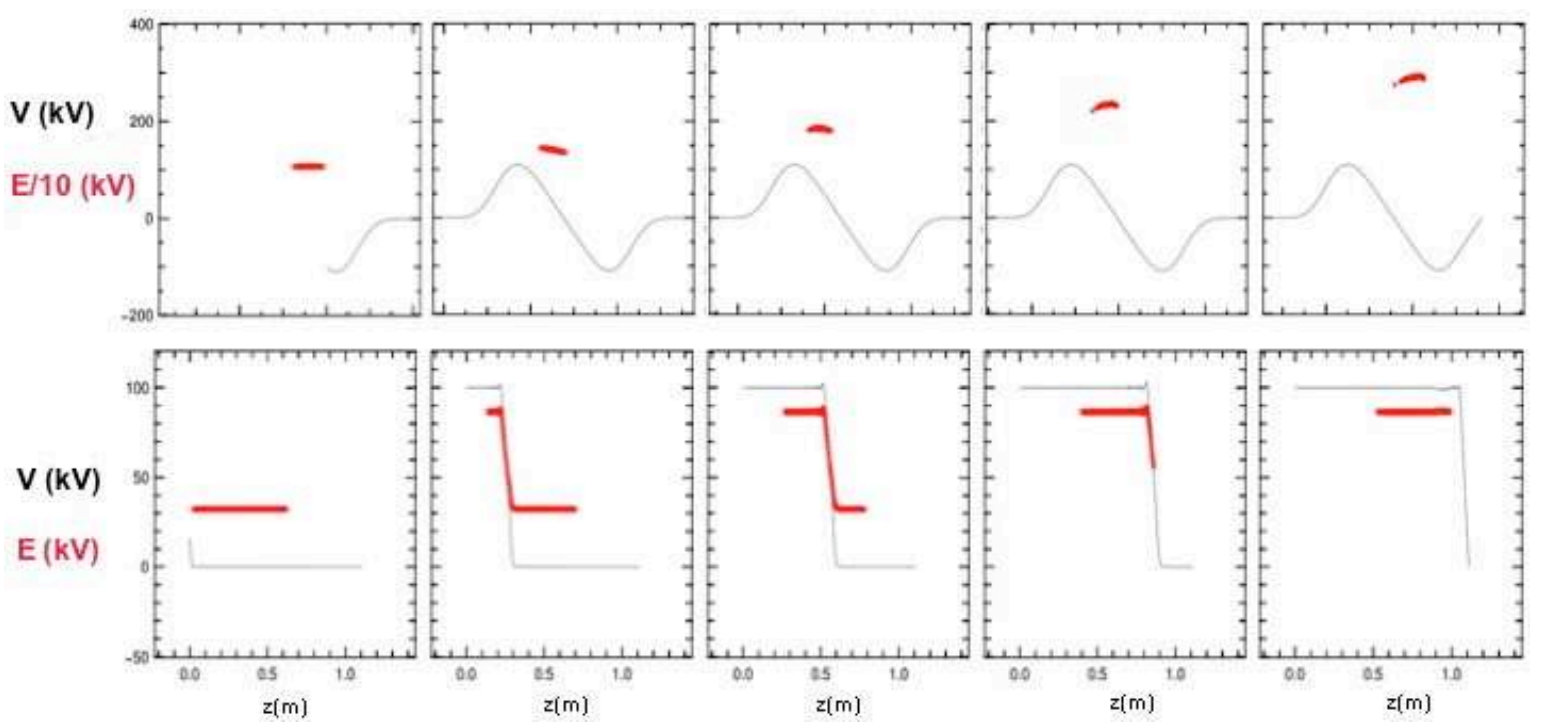

FIG. 3: Modes of PLIA operation. Upper row: short beam "surfs" on traveling voltage pulse (snapshots in wave frame); lower row: longer beam is accelerated by "snowplow" (snapshots in lab frame).

and mutual- inductances of the turns, as well as "loading" of the helix voltage by the beam current. At the long wavelengths of interest, there is very little Bessel function falloff of the accelerating field toward the axis; thus a simple code modeling longitudinal dynamics is useful for rough scoping. The two principal operating modes are illustrated via such a model in Fig. 3; these are the short-pulse ("surfing") mode appropriate to further acceleration of an established beam, and the long pulse ("snowplow") mode suitable for launching a slow-moving beam with possible pulse compression.

WARP simulations of the PLIA use a simplified circuit model tuned to obtain the correct circuit speed; the helix current and voltage are used as source terms in Poisson solvers to obtain the $\mathbf{E}$ and $\mathbf{B}$ fields. The runs clarify beam dynamics under the influence of space charge and circuit loading, and play a key role in experiment design. See [17]. Electromagnetic modeling and theoretical analysis is also underway [18, 19]. An improved "first principles" model, which accounts for all mutual capacitances and inductances and can capture end effects, transformer coupling, and wave dispersion, was formulated [20] and is planned for use in WARP.

In initial PLIA experiments on the NDCX apparatus [21], the beam energy was modulated by a ringing waveform and measured by an energy analyzer. A PLIA input voltage ranging from $-21 \mathrm{kV}$ to $+12 \mathrm{kV}$ yielded a beam energy modulation ranging from $-80 \mathrm{keV}$ to $+150 \mathrm{keV}$. WARP simulations, in which the helix voltage was measured at the system exit and advected backward at the wave speed to obtain the fields used to 

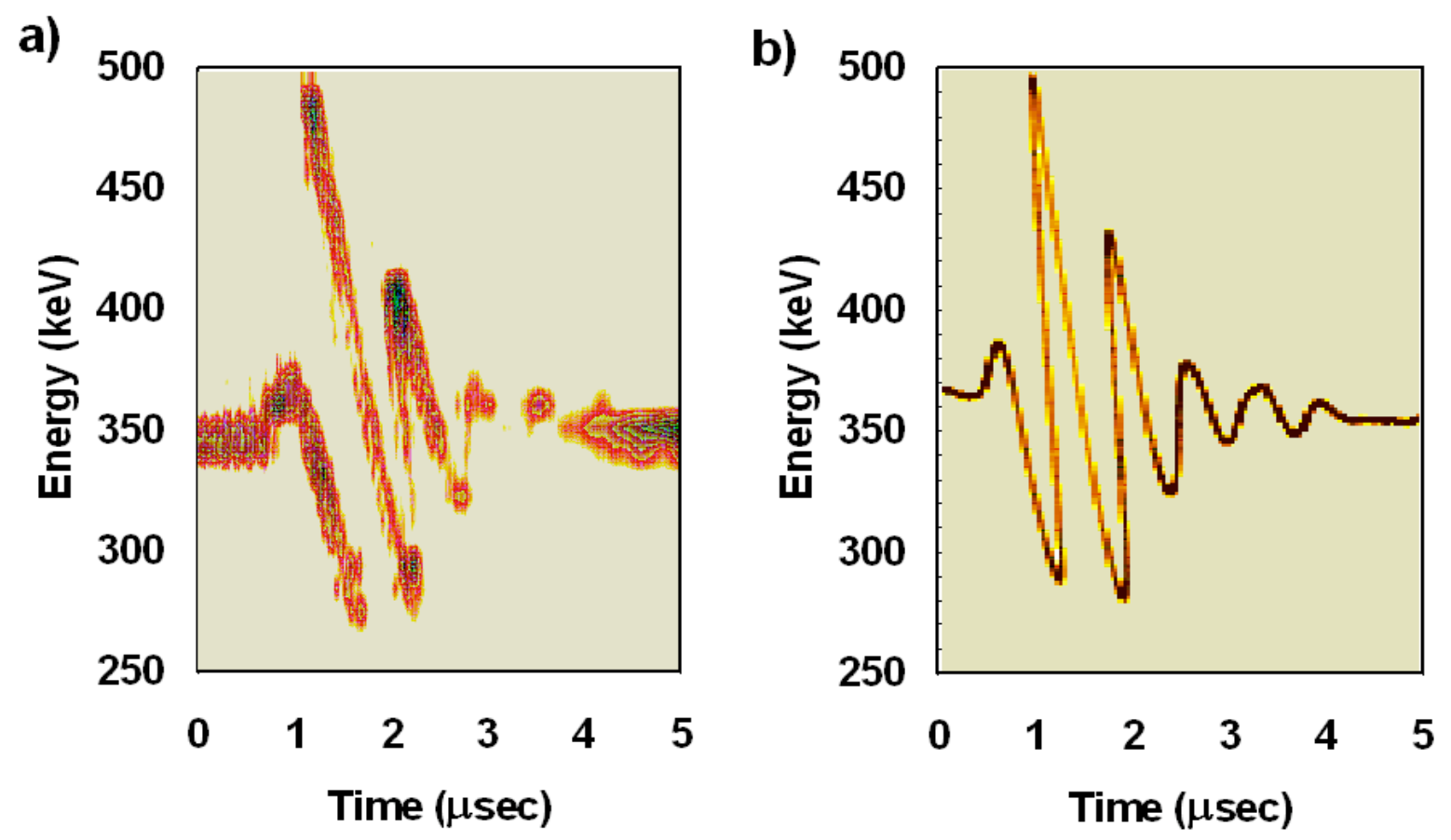

FIG. 4: Measured and simulated energy modulation in PLIA test.

advance the particles, are in rough agreement with the measurements (Fig. 4).

The oil dielectric / glass insulator helix used on these first tests had surface flashover problems at a few $\mathrm{kV} / \mathrm{cm}$; modified configurations are being tested [21]. To understand the breakdown, we are using WARP to follow "tracer" electrons emitted from the insulator surface in regions where the electric field has a radially-outward component. In the runs made to date, the PLIA fields are (assuming a dispersionless wave) "frozen" in the wave frame. Figure 5 shows the tracer paths in the wave frame after 135 ns. "Dangerous" electrons are deemed those that undergo modest energy gain and impact the insulator at points where further emission can occur.

\section{Electron Cloud and Gas Physics}

Positively-charged particle beams can suffer degradation due to the effects of stray particles, especially electron "clouds" (e-clouds) and gas. Experiments in the VNL have targeted this area as a major element of a campaign to understand the highbrightness transport of beams $[22,23]$. Much recent effort has been devoted to making the simulation tools more capable of supporting these efforts. Four developments are of note:

WARP/Posinst: WARP has been merged with the Posinst code [24], which provides 

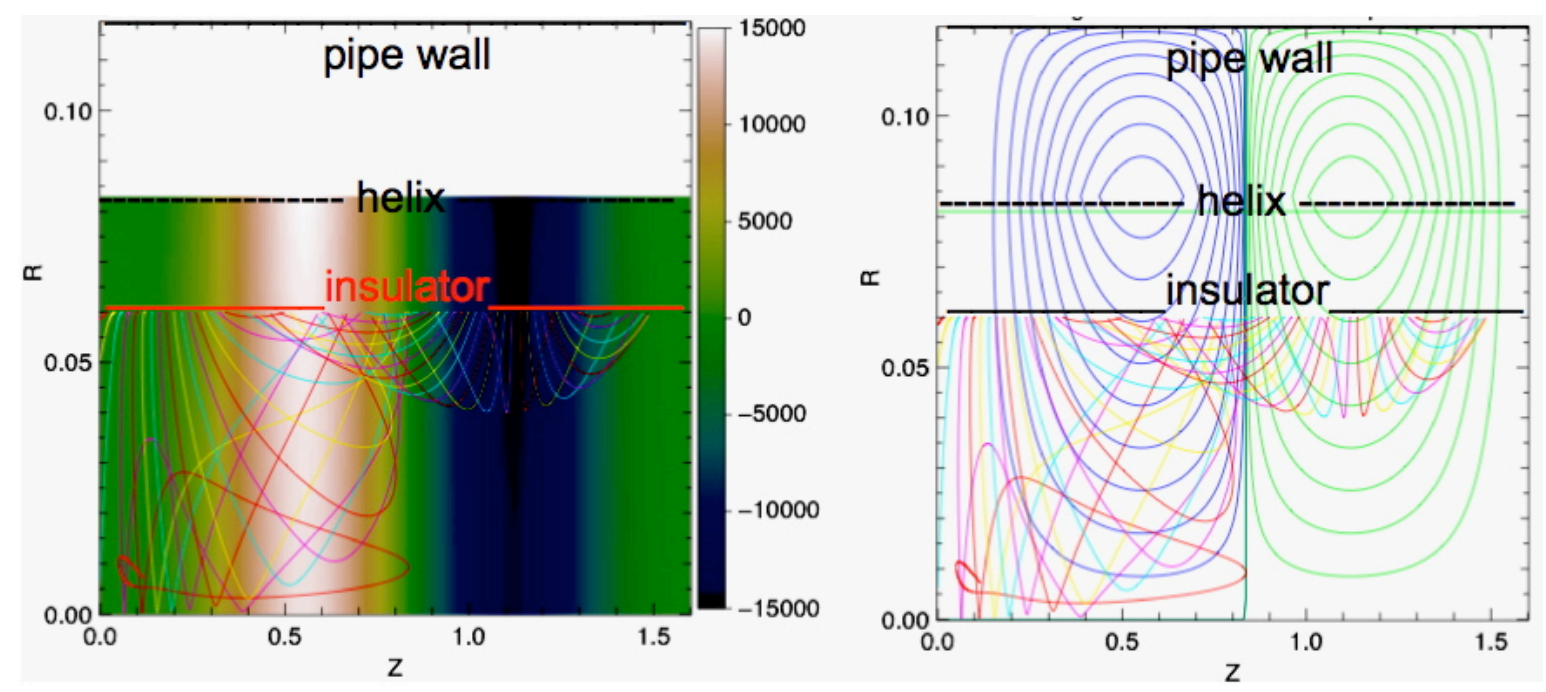

FIG. 5: Tracer electron paths in PLIA as computed by WARP, superposed on (left) electrostatic potential as color-coded and (right) magnetic field lines.

a model for electron-induced secondary electron emission.

Adaptive mesh refinement (AMR): HIFS-VNL staff pioneered in the integration of this widely-used technique with Particle-In-Cell (PIC) methods. This required analysis and development to minimize non-physical self-forces [25]. Further improvement of WARPs AMR-PIC simulation capabilities has led to a mature capability that is used routinely in both $3-\mathrm{D}$ and axisymmetric $(\mathrm{r}, \mathrm{z})$ geometries. This has led to large speedups, and has proven essential to simulations of beam injectors as well as of electron clouds and gas.

Multi-species interaction models: A comprehensive set of models governing the interaction of positively-charged beams with stray particles was developed and implemented in WARP $[22,23,26,27]$. Secondary electron emission induced by ions, neutral emission, and other processes are included. The TxPhysics package (Tech-X Corp.) [28] encapsulates several important plasma-wall interaction physics effects.

Large time-step particle advance: For self-consistent simulations including electron motion, a "mover" that interpolates between full particle dynamics and drift motion was developed. It enables time-steps much larger than the electron gyro-period (constrained instead by the next-larger timescale, which in a magnetic quadrupole is the electron bounce time in the electrostatic well), and thereby offers a computer-time reduction of 1-2 orders of magnitude [29, 30].

These capabilities have been applied to experiments on $\operatorname{HCX}[22,23,30]$, wherein the ion beam is directed onto a plate at the end of the system, generating copious primary electrons, which then generate secondaries. A set of control electrodes set to 
potentials that are varied from shot to shot offers a variety of operating conditions. The system has been modeled in considerable detail, with (in general) excellent agreement between data and code results. It is rich in phenomenology, including oscillations in the voltage measured on the electrodes. These studies are serving to benchmark both the codes and diagnostic techniques, and are also highly relevant to other systems involving positively-charged beams at high line charge densities. They have yielded such novel results as the first quantitative absolute measurement of electron cloud density. The effect on the beam is also predicted by the code, and good agreement is obtained when the initial beam distribution is obtained by "synthesizing" an initial distribution from experimental data using a reconstruction technique [1, 31]. WARP/Posinst is also being applied to other systems, including the Large Hadron Collider, and is playing a role in the VNL's experiments with solenoids, as described below.

\section{Solenoid Transport}

Transverse beam confinement via multi-beam quadrupole arrays has long been the baseline approach for the Inertial Fusion Energy (IFE) mission. However, transport using solenoids scales well to high line charge densities and low kinetic energies, and is currently preferred for the HEDP mission. Analysis and simulations have been clarifying this physics [32] and were applied to the design and interpretion of the STX [33]. Alignment requirements in solenoid systems have been assessed via WARP runs [34]. Experience on the Long Solenoid Experiment at the University of Maryland has lent useful guidance [35].

The STX beam often degrades when intercepted by a slit plate; the phenomenon coincides with high-frequency current oscillations on the electron trap. WARP $(\mathrm{r}, \mathrm{z})$ simulations show qualitatively similar oscillations [36]. It is believed that desorbed $\mathrm{H}_{2}$ gas near the plate is ionized by the beam, and the plasma buildup leads to a phenomenon akin to an oscillating virtual cathode. The oscillating potential pushes electrons through the trap into the solenoids, where their space charge affects the beam. See Fig. 6. 

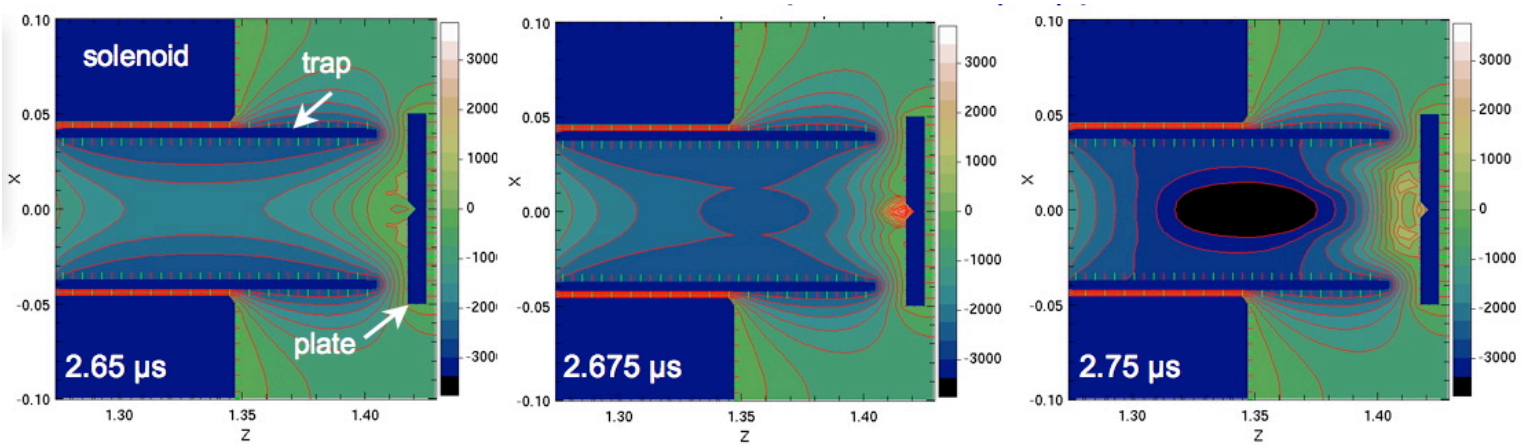

FIG. 6: Snapshots from a WARP simulation of STX with slit plate at end: contours of electrostatic potential $\phi(x, z)$, showing depression due to electrons.

\section{E. Beam and Plasma Injectors}

Merging-beamlet injector: The experiments on the $500-\mathrm{kV}$ test stand at LLNL (STS-500) successfully validated the multibeamlet injector concept, producing highbrightness, high-current beams using a very compact injector. This is especially important in the context of multiple-beam accelerators, where the expense and size of conventional injectors may impact the cost and beam quality. High-gradient tests with parallel beamlets were carried out, as were reduced-gradient scaled tests of a converging-beamlets system. WARP simulations were used to design the experiments, and results are in good agreement with those predictions [37-39].

High line-charge injectors: Several front-end approaches are possible; the "acceldecel" concept, which begins compressing the beam as it is injected, has received recent study [17], as has use of a PLIA in snowplow mode [40]. WARP simulations show that longitudinal space charge in such an injector can be controlled by shaping the waveform applied to the PLIA; see Fig. 7 [41]. 


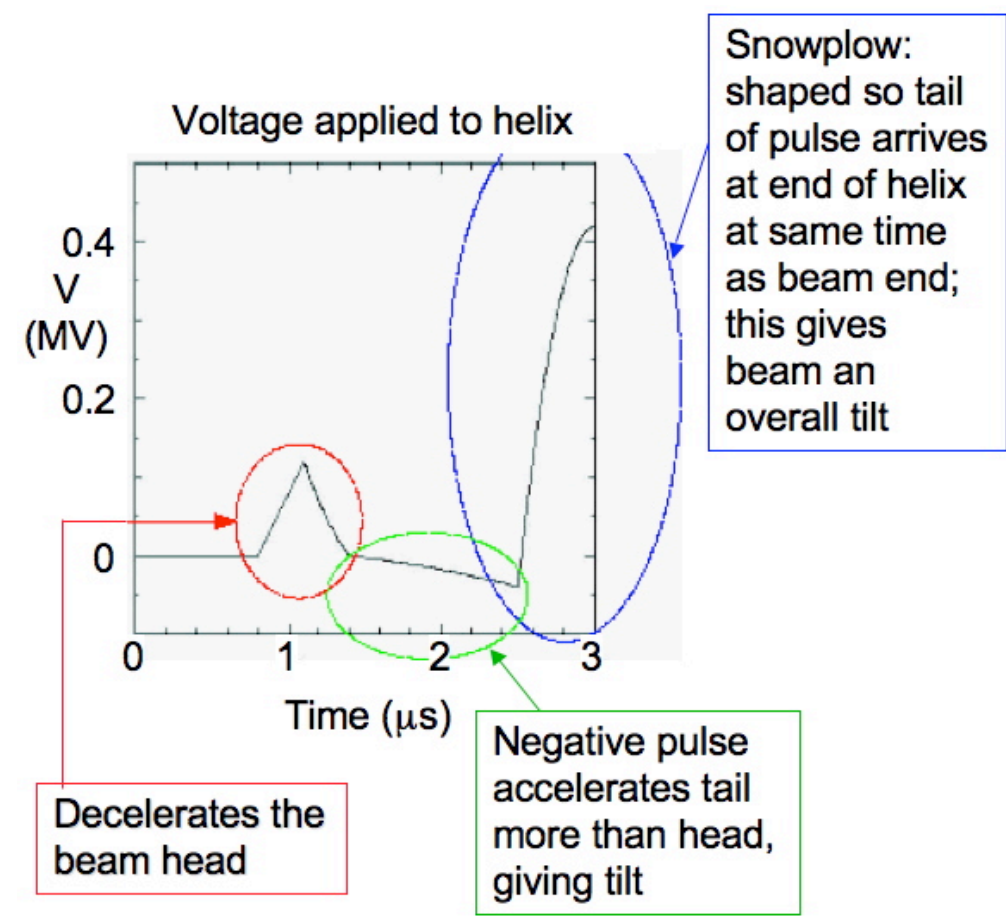

Axial profiles at $3.05 \mu \mathrm{s}$; beam is compressing due to velocity tilt

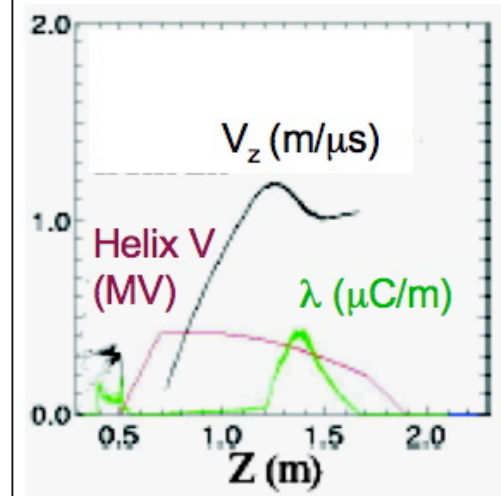

FIG. 7: Illustration of waveform used to control longitudinal space-charge in a high linecharge injector based on a PLIA in snowplow mode, and (on right) profiles of key quantities.

Plasma injectors: In order to achieve optimal pulse compressions, it is essential to produce a sufficiently dense plasma in the beam line. In support of the experiments, the behavior of the plasma in a ferroelectric plasma source was modeled in 3D using LSP, as was the evolution of the plasma density in a MEVVA magnetic field geometry. In one scenario, the coupling efficiency of plasma from the source to the region through which the beam is to drift is $\sim 50 \%$ [11].

\section{FUNDAMENTAL BEAM PHYSICS}

Beam transport limits: Experiments and simulations studying space-chargedominated beams in quadrupole transport channels show significant emittance growth and particle loss when the undepressed phase advance per lattice period exceeds about $85^{\circ}$. This limit has been employed in the design of accelerators for Heavy Ion Fusion without an understanding of its origin. Recent extensive particle-in-cell and coreparticle model runs have clarified the parametric dependence of the space charge limit and identified the processes responsible. This work is lending insight to present-day experiments and will be important for the design of future machines [42]. 
Neutralizing behavior of a background plasma: An analytical electron fluid model describing the plasma response to a propagating ion beam was developed. It predicts good neutralization when the beam pulse duration is much longer than the electron plasma period. In the opposite limit, the beam excites large-amplitude plasma waves, and if the beam density is larger than the background plasma density these waves break. The results agree well with particle simulations. See [43].

Two-stream instability: The electrostatic two-stream instability for a cold, longitudinally-compressing ion beam propagating through a background plasma has been investigated both analytically and numerically. It was found that the longitudinal beam compression leads to a significant reduction in the growth rate of the two-stream instability compared to the case without an initial velocity tilt [44-46].

Anisotropy-driven instabilities: In a magnetized plasma, the electrostatic Harris instability appears when the transverse temperature sufficiently exceeds the longitudinal temperature. In an intense beam, betatron motion can substitute for cyclotron motion, and a Harris-like instability appears. The mode was observed in WARP runs, identified as a possible issue [47], and explored systematically [48]. Detailed understanding has come from BEST $\delta$ f simulations and analysis. During the nonlinear stage of the instability, the particle velocities are randomly scattered by the many waves that have been excited, which leads to a quasilinear mixing in phase space and eventual longitudinal thermalization well before the temperatures are equalized [49]. The electromagnetic Weibel instability, also driven by anisotropy, has been studied [44, 50]; a Darwin model based on the canonical momentum formulation is being implemented in BEST to facilitate further studies of this mode.

Dynamics of bunched beams: When the bunch length is compressed by a large factor, the coupling between the longitudinal and transverse dynamics induced by the 3D nonlinear space-charge fields become significant. A consequence is that the particle dynamics does not conserve transverse energy and longitudinal energy separately, and there exists no exact kinetic equilibrium with anisotropic temperature. A reference state for beams with anisotropic temperature was derived; it is not an exact equilibrium. The difference between the exact (time dependent) distribution and the reference state is simulated via a generalized $\delta \mathrm{f}$ particle simulation algorithm. If the beam temperature is isotropic, the reference state is an exact equilibrium, and the generalized algorithm reduces to the conventional one. Even in this case, particle trajectories on constant energy surfaces are non-integrable, so that it is impossible to perform an integration along unperturbed orbits to analytically calculate the linear eigenmodes [44, 51].

\section{OTHER APPLICATIONS AND NEW CAPABILITIES}

WARP has long been a key tool for understanding of the University of Maryland Electron Ring (UMER); see [35]. Among the noteworthy results obtained, virtual cathode oscillations predicted by WARP (with mesh refinement) were later observed 
$([52])$.

WARP is also used to model non-neutral particle traps, including the Princeton Paul Trap experiment (PTSX), which models quadrupole confinement of intense beam ([53]). More recently, the code has been applied to the UC Berkeley Penning-Malmberg trap, which includes a multipole field (necessary for anti-Hydrogen confinement) [30, 54].

Other new capabilities are of note. A new "one pass" algorithm was developed and implemented in LSP for implicit solution of the electromagnetic field equations [55]. BEST was optimized for massively parallel computers and applied to collective effects of 3D bunched beams and the temperature-anisotropy instability. Improved beam initializations were added to WARP (as part of a formal collaboration between the US and Japan), adding the ability to load a wide variety of self-consistent initial distribution functions. "Subcyling" was implemented in WARP, adding the ability to advance different groups of particles with different timesteps, self-consistently. Finally, a new equation-of-state formulation was developed for LSP, consistent with the code's particle-based fluid model.

\section{FUTURE DIRECTIONS}

The needs of the Heavy ion Fusion Science program will continue to set the directions for beam modeling and code development. The near term goal is to develop drivers for Warm Dense Matter at $\sim 1 \mathrm{eV}$, at minimal cost. This will involve combining bunch compression with transverse focusing, as planned for the next stage of the experiments. A successful system must account for beam energy spread (chromatic aberrations) and must achieve the necessary "contrast ratio" (by avoiding excessive prepulse on target). The new emphasis on solenoids will require further studies of both beam dynamics (we plan to extend the study of beam transport limits in quadrupoles, described herein, to solenoids) and electron cloud effects; this should include a comparative assessment of such effects in solenoid-confined and magnetic quadrupole-confined beams. Another near term priority will be to eliminate the flashover in the PLIA, with the help of further WARP modeling.

In the longer term, it will be important to develop an improved path to IFE, building on the new ideas currently being explored. In one new concept, based on a modular multi-pulse driver, pulses overtake each other and overlap at the target. Such a machine might be based on either induction or PLIA accelerating sections. The approach would offer a modular development path. It will also be desirable to explore other new or rediscovered ideas, such as accelerator-driven fast ignition. A critical assessment of these and other new ideas, and a selection of the most promising for further study, will be needed. Here, an enhanced "systems" model would be helpful.

The anticipated greater availability of time on massively parallel computers can be expected to open the path to accurate end-to-end simulations of a driver-scale accelerator. For this to happen, the codes need to be validated on near-term experiments, 
and modified for use on terascale or larger computers. The improvements will require algorithmic changes and enhancements to such capabilities as restart files and diagnostic output, which can become challenging when tens of thousands of processors are used.

A number of developments may be anticipated. The major codes (WARP, LSP, and BEST) will continue to be exploited, and improved as needed. It will be important to build on WARP's new capabilities (especially the e-cloud models, large-timestep electron mover, adaptive mesh refinement, and subcycling), by generalizing the code to efficiently handle higher plasma densities. The methods will differ from those used in LSP, enabling cross-checking. Source-through-target modeling will be pursued using multiple approaches, and with an eye toward massively parallel computations. Because of the potential importance of the PLIA architecture, an improved PLIA model will be implemented in WARP, including end effects, transformer coupling, and realistic dispersion. Finally, it will be important to develop improved numerical methods for the quantitative prediction of beam halo production and loss rates, in both quadrupole and solenoid systems. Vlasov methods are making rapid progress, and may open the door to doing this efficiently [56].

\section{Acknowledgments}

The work of a number of people formed the basis for this paper; the References provide a partial list. This work was performed under the auspices of the U.S. D.O.E. by the University of California, Lawrence Livermore National Laboratory under Contract No. W-7405-ENG-48.

[1] A. Friedman, "Simulation of intense beams for Heavy Ion Fusion," Nucl. Instr. and Meth. A 544 (2005) 160.

[2] B. G. Logan, et al., "Progress in the Heavy Ion Fusion Sciences Virtual National Laboratory," Nucl. Instr. and Meth., these proceedings.

[3] J. J. Barnard, et al., "Theory and simulation of warm dense matter targets," Nucl. Instr. and Meth., these proceedings.

[4] W. M. Sharp, et al., "Simulation of Drift-Compression for Heavy-Ion-Fusion," Nucl. Inst. and Meth. A 544 (2005) 398.

[5] P. K. Roy, et al., "Neutralized Drift Compression Experiments (NDCX) with a High Intensity Ion Beam," Nucl. Instr. and Meth., these proceedings. 
[6] P. A. Seidl, et al., "Plans for longitudinal and transverse neutralized beam compression experiments and initial results from solenoid transport," Nucl. Instr. and Meth., these proceedings.

[7] A. B. Sefkow, et al., "A Fast Faraday Cup to Measure Neutralized Drift Compression in Intense Ion Charge Bunches," Phys. Rev. Special Topics Accel. Beams 9 (2006) 052801.

[8] A. B. Sefkow and R. C. Davidson, "Theoretical Models for Describing Longitudinal Bunch Compression in the Neutralized Drift Compression Experiment," submitted for publication (2006) to Phys. Rev. Special Topics Accel. Beams.

[9] C. Thoma, et al., "Comparison of experimental data and three-dimensional simulations of ion beam neutralization from the Neutralized Transport Experiment," Phys. Plasmas 12 (2005) 043102.

[10] E. Henestroza, et al., "Design and Characterization of a Neutralized-Transport Experiment for Heavy-Ion Fusion," Phys. Rev. Special Topics Accel. Beams 7 (2004) 083501.

[11] A. Sefkow, et al., "Optimized Simultaneous Transverse and Longitudinal Focusing of Intense Ion Beam Pulses for Warm Dense Matter Applications," Nucl. Instr. and Meth., these proceedings.

[12] D. R. Welch, et al., "Integrated Simulation of an Ion-Driven Warm Matter Experiment," Nucl. Instr. and Meth., these proceedings.

[13] H. Qin, et al., "Drift Compression and Final Focus Options for Heavy Ion Fusion," Nucl. Instr. and Meth. A, 544 (2005) 255.

[14] Proc. Workshop on Accel. Driven High Energy Density Physics, LBNL, Oct. 26-9, 2004, J. J. Barnard, Ed.; http://hifweb.lbl.gov/public/hedpworkshop .

[15] A. Friedman, et al., "Highly compressed ion beams for High Energy Density Science," paper ROAB003, IEEE / APS Part. Accel. Conf. (Knoxville, May 2005), http://accelconf.web.cern.ch/accelconf/ .

[16] R. J. Briggs, "Pulse line ion accelerator concept," Phys. Rev. ST Accel. Beams 9 (2006) 060401.

[17] E. Henestroza, et al., "Extraction and Compression of High Line Charge Density Ion Beams," Proc. 2005 Particle Accelerator Conf., paper FPAT028, http://accelconf.web.cern.ch/accelconf/ .

[18] S. D. Nelson, et al., "Electromagnetic Simulations of Helical-based Ion Acceleration Structures," Proc. 2005 Particle Accelerator Conf., paper FPAT037, http://accelconf.web.cern.ch/accelconf/ .

[19] G. Caporaso, et al., "Dispersion Analysis of the Pulseline Accelerator," Proc. 2005 Particle Accelerator Conf., paper FPAT034, http://accelconf.web.cern.ch/accelconf/ .

[20] A. Friedman, "Solving the quasi-static field model of the pulse-line accelerator; relationship to a circuit model," LLNL Report UCRL-TR-218776 (2006).

[21] J. E. Coleman, et al., "Low Voltage Beam Experiments on the Pulse Line Ion Acceler- 
ator," Nucl. Instr. and Meth., these proceedings.

[22] A. W. Molvik, et al., "Quantitative electron and gas cloud experiments," Nucl. Instr. and Meth., these proceedings.

[23] J.-L. Vay, et al., "Self-consistent simulations of heavy-ion beams interacting with electron-clouds," Nucl. Instr. and Meth., these proceedings.

[24] M. A. Furman and M. Pivi, "Probabilistic model for the simulation of secondary electron emission," Phys. Rev. ST Accel. Beams 5 (2002) 124404.

[25] J-L. Vay, et al., "Application of Adaptive Mesh Refinement to Particle-In-Cell Simulations of Plasmas and Beams," Phys. Plasmas 11 (2004) 2928.

[26] J-L. Vay, et al., "Filling in the Roadmap for Self-Consistent Electron Cloud and Gas Modeling," IEEE / APS Part. Accel. Conf. (Knoxville, May 2005), http://accelconf.web.cern.ch/accelconf/

[27] P. Stoltz, et al., "Cross-platform, multi-language physics modules for heavy ion simulation," Nucl. Instr. and Meth., these proceedings.

[28] http://www.txcorp.com/technologies/TxPhysics

[29] R. H. Cohen, et al., "Simulating Electron Clouds in Heavy Ion Beams," Phys. Plasmas $12(2005) 056708$.

[30] R. H. Cohen, A. Friedman, D.P. Grote, and J.-L. Vay, "Large-timestep mover for particle simulations of arbitrarily magnetized species," Nucl. Instr. and Meth., these proceedings.

[31] A. Friedman, D.P. Grote, C.M. Celata, J.W. Staples, "Use of projectional phase space data to infer a 4D particle distribution," Laser and Particle Beams 21 (2003) 17.

[32] E. P. Lee, "Solenoid Transport for Heavy Ion Fusion," Nucl. Inst. Meth. A 544 (2005) 187.

[33] E. Henestroza, et al., "Solenoid Transport and Final Focusing System for High Intensity Ion Beams," Nucl. Instr. and Meth., these proceedings.

[34] I. Haber, private communication, Dec. 2005.

[35] I. Haber, et al., "Scaled Electron Experiments at the University of Maryland," Nucl. Instr. and Meth., these proceedings.

[36] W. M. Sharp, et al., "Simulating Electron Clouds in High-Current Ion Accelerators with Solenoid Focusing," Nucl. Instr. and Meth., these proceedings.

[37] D. P. Grote, E. Henestroza, and J. W. Kwan, "Design and Simulation of a Multibeamlet Injector for a High Current Accelerator," Phys. Rev. Special Topics Accel. Beams 6 (2003) 014202.

[38] D. P. Grote, "Design and Modeling of the Multibeamlet Injector," Nucl. Instr. and Meth., these proceedings.

[39] G. A. Westenskow, D. P. Grote, J. W. Kwan, and F. M. Bieniosek, "High-Brightness Heavy-Ion Injector Experiments," Nucl. Instr. and Meth., these proceedings.

[40] E. Henestroza, J. J. Barnard, J. W. Kwan, and S. S. Yu, "High Brightness Injector for Warm Dense Matter Studies," Nucl. Instr. and Meth., these proceedings. 
[41] D. P. Grote, private communication (2006).

[42] S. M. Lund, S. R. Chawla, and S. H. Chilton, "Space Charge Transport Limits of Ion Beams in Periodic Quadrupole Focusing Channels," Nucl. Instr. and Meth., these proceedings.

[43] I.D. Kaganovich, et al., "Effects of Finite Pulse Length, Electron Temperature, Solenoidal Magnetic Field, and Gas Ionization on Ion Beam Pulse Neutralization by Background Plasma," Nucl. Instr. and Meth., these proceedings.

[44] R. C. Davidson, et al., "Collective Interaction Processes in Intense Heavy Ion BeamPlasma Systems," Nucl. Instr. and Meth., these proceedings.

[45] E. A. Startsev and R. C. Davidson, "Dynamic Stabilization of Two-Stream Instability During Longitudinal Compression of Intense Charged Particle Beam Propagation Through Background Plasma," Nucl. Instr. and Meth., these proceedings.

[46] E. A. Startsev and R. C. Davidson, "Two-Stream Instability for a LongitudinallyCompressing Charged Particle Beam," Phys. Plasmas 13 (2006) 062108.

[47] A. Friedman, D. P. Grote, and I. Haber, "Three-dimensional particle simulation of heavyion fusion beams," Phys. Fluids B 4 (1992) 2203.

[48] I. Haber, D. A. Callahan, A. Friedman, D. P. Grote and A. B. Langdon, "Transverselongitudinal temperature equilibration in a long uniform beam," Fusion Engineering and Design 32-33 (1996) 169.

[49] E. A. Startsev, R. C. Davidson and H. Qin, "Anisotropy-driven collective instability in intense charged particle beams", Phys. Rev. ST Accel. Beams 8 (2005) 124201.

[50] R. C. Davidson, E. A. Startsev, I. D. Kaganovich and H. Qin, "Multispecies Weibel and Two-Stream Instabilities for Intense Ion Beam Propagation Through Background Plasma," IEEE / APS Part. Accel. Conf. (Knoxville, May 2005), http://accelconf.web.cern.ch/accelconf/

[51] H. Qin, R. C. Davidson, and E. A. Startsev, "Nonlinear Pertubative Simulation Studies of Collective Processes in 3D Finite-Length Charge Bunches at High Space-Charge Intensities," Nucl. Instr. and Meth., these proceedings.

[52] I. Haber, et al., "Measurement and Simulation of the Time-Dependent Behavior of the UMER Source," Nucl. Instr. and Meth., these proceedings.

[53] E. P. Gilson, et al., "Conditions for Minimization of Halo Particle Production During Transverse Compression of Intense Ion Charge Bunches in the Paul Trap Simulator Experiment (PTSX)," Nucl. Instr. and Meth., these proceedings.

[54] K. Gomberoff, et al., "Simulation studies of non-neutral plasma equilibria in an electrostatic trap with a magnetic mirror," in submission to Phys. Plasmas (2006).

[55] D. R. Welch, et al., "Implementation of an non-iterative implicit electromagnetic field solver for dense plasma simulation," Comp. Phys. Commun. 164 (2004) 183.

[56] M. Gutnic, G. Latu, and E. Sonnendrücker, "Adaptative two-dimensional Vlasov simulation of heavy ion beams," Nucl. Instr. and Meth., these proceedings. 